\title{
Resistencia de la soya [Glycine max. (L.) Merr.] al estrés por calor en estado de plántula
}

Soybean [Glycine max. (L.) Merr.] resistance to heat stress at seedling stage

García-Rodríguez Julio C. ${ }^{1 凶}$, Muñoz-Orozco Abel ${ }^{2}$, Maldonado-Moreno Nicólas ${ }^{1}$, Cruz-Izquierdo Serafín $^{2}$ y Ascencio-Luciano Guillermo ${ }^{1}$

${ }^{1}$ Campo Experimental Las Huastecas-INIFAP. Carretera Tampico-Mante km 55, Villa Cuauhtémoc, Altamira, Tamaulipas. C.P. 89610. Tel. 01-55-38718700, Ext. 83319. ${ }^{2}$ Colegio de Postgraduados, campus Montecillo. Carretera México-Texcoco km 36.5, Montecillo, Texcoco, Edo. de México. C.P. 56230. Tel. 01-595-9520200.

${ }^{\otimes}$ Autor para correspondencia: garcia.juliocesar@inifap.gob.mx

Recibido: 17/01/2016

Aceptado: 21/06/2016

\section{RESUMEN}

El objetivo del presente estudio fue conocer la respuesta de 21 genotipos de soya a altas temperaturas en estado de plántula. Se evaluaron cuatro niveles de temperatura: $\theta 1=50 \pm 1{ }^{\circ} \mathrm{C}, \theta 2=54 \pm 1{ }^{\circ} \mathrm{C}, \theta 3=$ $58 \pm 1{ }^{\circ} \mathrm{C}$ y $\theta 4=62 \pm 1{ }^{\circ} \mathrm{C}$, los cuales fueron acumulativos. Para $\theta 1$ se calificó el grado de cierre de las hojas $(\mathrm{GCH})$ y para el resto de los tratamientos el grado de marchitez (GM). Posterior al último tratamiento se aplicó un riego y se observó el grado de recuperación (GR) de las plántulas estresadas. Se utilizó un diseño completamente al azar desbalanceado para GCH y GR; en GM fue completamente al azar en arreglo factorial 21 x 3. Se observaron diferencias entre genotipos en $\mathrm{GCH}$ : seis fueron insensibles, mientras que H98-1240 cerró sus hojas por completo. Considerando los efectos genéricos, $\theta 4$ provocó el mayor GM y entre genotipos, 15 presentaron un bajo GM, destacando H02-1337 como el menos marchito; H98-1240 resultó el más afectado. Según los efectos específicos, H02-1337 no sufrió cambios al pasar de $\theta 2$ a $\theta 3$, considerándose el más resistente en este nivel; mientras que H02-2248 aumentó en menor proporción el GM entre $\theta 3$ y $\theta 4$. El GR posterior al riego indicó que los genotipos con mayor GM tardaron más en recuperarse, incluso algunos no lo hicieron y viceversa; sin embargo dicha variabilidad se debió probablemente a efectos aleatorios.

Palabras clave: Glycine max L. (Merr.), resistencia a calor, estrés abiótico, termotolerancia.

\begin{abstract}
The aim of this study was to determine the response of 21 soybean genotypes at seedling stage to high temperatures. Four temperature levels were evaluated: $\theta 1=50 \pm 1{ }^{\circ} \mathrm{C}, \theta 2=54 \pm 1{ }^{\circ} \mathrm{C}, \theta 3=58 \pm 1{ }^{\circ} \mathrm{C}$,
\end{abstract}


and $\theta 4=62 \pm 1{ }^{\circ} \mathrm{C}$, which were cumulative. Closure degree of leaves $(\mathrm{GCH})$ for $\theta 1$ and wilting degree (GM) for the rest of treatments were scored. After last treatment, irrigation was applied and recovery degree (GR) of stressed seedling was observed. Experimental design was completely random unbalanced for $\mathrm{GCH}$ and GR; for GM was completely randomized in factorial 21 x 3 arrangement. Differences in GCH were observed between genotypes: six were insensitive, while H98-1240 closed its leaves completely. Regarding generic effects, $\theta 4$ caused the largest GM and between genotypes, 15 have a low GM, H02-1337 excelled as the less wilted; H98-1240 was the most affected. According to specific effects, H02-1337 unchanged GM from $\theta 2$ to $\theta 3$, considered a resistant genotype at this level; while H02-2248 increased GM to a lesser extent between $\theta 3$ and $\theta 4$. The GR after irrigation indicated that the most GM genotypes took longer to recover, even some ones not recovered, and vice versa; however this variability it was due to random effects probably.

Key words: Glycine max L. (Merr.), heat resistance, abiotic stress, thermotolerance.

\section{INTRODUCCIÓN}

Las altas temperaturas pueden desencadenar una serie de cambios morfoanatómicos, fisiológicos y bioquímicos en las plantas, que afectan su crecimiento y desarrollo, repercutiendo en la reducción del rendimiento. El estrés por calor se define como un aumento en la temperatura, durante un periodo de tiempo suficiente para causar daños irreversibles en el crecimiento y desarrollo de las plantas. La elevación de la temperatura, por lo general de 10 a $15{ }^{\circ} \mathrm{C}$ por encima de la temperatura ambiente, es considerada como estrés. Sin embargo, el estrés por calor es un factor complejo que depende de la intensidad, la duración y la tasa de aumento de la temperatura (Wahid et al., 2007).

García y Muñoz (2013) ${ }^{1}$ utilizaron un modelo para el análisis de información meteorológica con datos históricos, con el fin de conocer el estatus del sur de Tamaulipas, región con mayor producción de soya en México, con respecto al cambio climático (datos no publicados). Los resultados elucidaron que los patrones de temperatura y precipitación están cambiando. La temperatura máxima se incrementó entre 1.5 y $2{ }^{\circ} \mathrm{C}$ aproximadamente en el periodo de 1976 a 2009, en relación con el periodo 1961 a 1975. Además se encontraron años atípicos donde se observaron golpes de calor por arriba de los 38 ${ }^{\circ} \mathrm{C}$.

De acuerdo con Wahid et al. (2007), los efectos del estrés por calor en la agricultura pueden ser mitigados mediante el desarrollo de cultivares con mejor termotolerancia. Tolerancia al calor es la capacidad de la planta para crecer y producir rendimiento económico en condiciones de altas temperaturas. Mientras que algunos investigadores piensan que las temperaturas nocturnas son las que limitan el desarrollo de las plantas, otros argumentan que las temperaturas del día y la noche no afectan de forma independiente, y que la temperatura diurna promedio es la mejor para caracterizar la respuesta de las plantas al incremento del calor (Peet y Willits, 1998).

Según Sullivan (1972), aunque la tolerancia al incremento en la temperatura normal involucra mecanismos complejos de resistencia, uno de los mecanismos principales es la termoestabilidad de la membrana celular (Martineau et al., 1979). A nivel molecular, la expresión de un grupo de proteínas, llamadas de choque térmico (HSP por sus siglas en inglés), juegan un rol importante en la adquisición de la 
termotolerancia en las plantas (Alexandrov, 1994).

En el caso de la soya, además de ser sensible al fotoperiodo, Allen y Boote (2000) reportaron que es sensible a la temperatura. La soya crece y se desarrolla con temperaturas óptimas de 25 a $30{ }^{\circ} \mathrm{C}$ (Raper y Kramer, 1987; Maldonado et al., 2013), pero entre los 33 y 40 ${ }^{\circ} \mathrm{C}$ reduce su crecimiento ( $\mathrm{Vu}$ et al., 1997). Algunos trabajos que evaluaron el efecto de la temperatura en el desarrollo de la soya, indicaron que el inicio de la floración se retrasó con temperaturas de $32{ }^{\circ} \mathrm{C}$ (Borthwick y Parker, 1940), que la formación de la semilla tardó más con $30-40{ }^{\circ} \mathrm{C}$ (Thomas et al., 2003), y que cuando las plantas fueron expuestas a 35 ${ }^{\circ} \mathrm{C}$ por 10 h durante el día, hubo disminución en el rendimiento de aproximadamente $27 \%$ (Gibson y Mullen, 1996).

Por otro lado, utilizando una técnica de selección basada en la viabilidad del polen a altas temperaturas, Mohammed et al., (2007) diferenciaron genotipos de soya: tolerantes, con tolerancia intermedia y sensibles al calor, además se reveló que la termoestabilidad de la membrana no se correlacionó con los parámetros del polen y que la tolerancia de los tejidos vegetativos tiene poca o nula relación con la tolerancia de los tejidos reproductivos.

Lobell y Asner (2003) evaluaron la relación entre la variación del clima y la producción de maíz y de soya en USA entre 1982 y 1998 , reportando que por cada $1{ }^{\circ} \mathrm{C}$ de aumento en la temperatura, hubo una disminución promedio de $17 \%$ en el rendimiento. También, germoplasma de soya de los grupos de madurez III al VII fue evaluado en tres niveles de potencial osmótico y cuatro regímenes de temperatura para tolerancia a sequía y calor respectivamente, encontrándose que los daños más severos por calor se dieron durante la floración, que la susceptibilidad a dicho factor decreció hacia la madurez y que hubo gran variabilidad entre líneas en cuanto a la tolerancia, tanto para sequía como para temperaturas extremas, pero una no se correlacionó con la otra (Sapra y Anaele, 1991).

El propósito de este trabajo consistió en evaluar la respuesta de 21 genotipos de soya a altas temperaturas durante el estado de plántula, mediante la inyección de aire caliente, en condiciones de invernadero.

\section{MATERIALES Y ÉTODOS}

El experimento se desarrolló bajo condiciones de invernadero en el Colegio de Postgraduados, campus Montecillo, Texcoco, Edo. de México. La siembra se realizó el 15 de octubre de 2013 en vasos desechables de plástico del número 8 . Se utilizaron $300 \mathrm{~g}$ de suelo vaso $^{-1}$, en donde se depositaron dos semillas de soya. Posterior a la emergencia se aclaró a una plántula vaso ${ }^{-1}$.

Se aplicaron tres riegos a capacidad de campo, uno inmediatamente después de la siembra y dos antes de someter las plántulas a los tratamientos de calor, con una separación de siete días entre cada uno aproximadamente. Las plántulas se sometieron al tratamiento de calor a los 15 días después de la siembra, cuando tenían las primeras hojas verdaderas (unifoliadas).

Los genotipos (G) evaluados correspondieron a las variedades: Huasteca 200, Huasteca 300 y Tamesí, generadas por el INIFAP para el trópico húmedo de México, y las líneas experimentales: H98-1052, H021656, H02-2309, H02-1337, H98-1325, H981240, H98-1021, H02-2248, H02-2091, H022331, H02-2295, H02-1991, H02-1123, H022224, H06-1192, H06-0698, H10-0242 у H100556, del programa de mejoramiento genético de soya del mismo Instituto.

Se evaluaron cuatro niveles de temperatura que fueron acumulativos: $\theta 1=50$ $\pm 1{ }^{\circ} \mathrm{C}, \theta 2=54 \pm 1{ }^{\circ} \mathrm{C}, \theta 3=58 \pm 1{ }^{\circ} \mathrm{C}$ y $\theta 4=$ $62 \pm 1{ }^{\circ} \mathrm{C}$, todos por periodos de dos horas. Los 
niveles de $\theta$ se aplicaron con una separación de 24 h entre cada uno sobre las mismas plántulas.

En $\theta 1$ se calificó el grado de cierre de las hojas $(\mathrm{GCH})$, ya que este comportamiento, denominado movimiento nástico, es típico de varias especies vegetales por efecto de un estímulo externo que puede ser iluminación, falta de agua, temperatura, etc. La escala utilizada fue del 1 al 5, donde 1 significó posición totalmente abierta de las hojas y 5 posición totalmente cerrada de las hojas (paralela con el tallo). En el resto de $\operatorname{los} \theta$, se registró el grado de marchitez (GM) también en una escala de 1 a 5 , donde 1 correspondió a nulo o leve marchitamiento y 5 a marchitamiento avanzado o muerte total del tejido vegetal. Después del último tratamiento se regaron las plántulas a capacidad de campo y a los tres días se observó el grado de recuperación (GR) utilizando la misma escala, pero en este caso 1 fue para recuperación alta y 5 para recuperación baja.

Para la aplicación de los tratamientos se diseñó una cámara de madera con flujo de calor por medio de un dispositivo generador de aire caliente, con capacidad para regular de manera manual la temperatura y la velocidad del aire, alcanzando niveles de temperatura por arriba de los $60{ }^{\circ} \mathrm{C}$. A la cámara se le acondicionó un mecanismo que permitió que el aire caliente no golpeara directamente a las plántulas. El monitoreo de la temperatura se realizó colocando seis termómetros de inmersión, dos en cada uno de los lados de la cámara, aproximadamente a la altura del follaje.
Se utilizó un diseño completamente al azar desbalanceado para el análisis de $\mathrm{GCH}$ en $\theta 1$ y GR posterior al riego después del $\theta 4$. El GM en el resto de los tratamientos se analizó como un diseño completamente al azar con arreglo factorial $21 \times 3$. El análisis de varianza y la comparación de medias (Duncan, $\mathrm{p} \leq 0.05$ ) se efectuaron mediante el programa SAS (SAS Institute, 2009). Además se calculó el coeficiente de correlación de Pearson para conocer el grado de asociación entre las variables. En todos los casos se utilizaron datos transformados por raíz cuadrada. La selección de genotipos con mejor proporción de efectos genéricos y específicos al estrés por calor, se realizó adaptando el modelo uno de resistencia a sequía, publicado por Muñoz (1992).

\section{RESULTADOS Y DISCUSIÓN}

Los resultados del análisis de variación para $\mathrm{GCH}$ en $\theta 1$ mostraron efectos significativos $(\mathrm{p} \leq 0.05)$ entre genotipos, diferenciándose claramente un grupo: H021656, H02-2309, H98-1325, Huasteca 300, H06-0698 y H10-0556, que fue totalmente insensible a la aplicación de $50 \pm 1{ }^{\circ} \mathrm{C}$ de temperatura (Figura 1), manteniendo sus hojas totalmente abiertas. Mientras tanto, H98-1240 fue el más afectado alcanzando el GCH más alto (Figura 1). Cabe señalar que $\theta 1$ no generó indicios de marchitamiento (desecación) sobre los genotipos, denotando la capacidad de resistencia de la soya a temperaturas por arriba de su umbral térmico. 


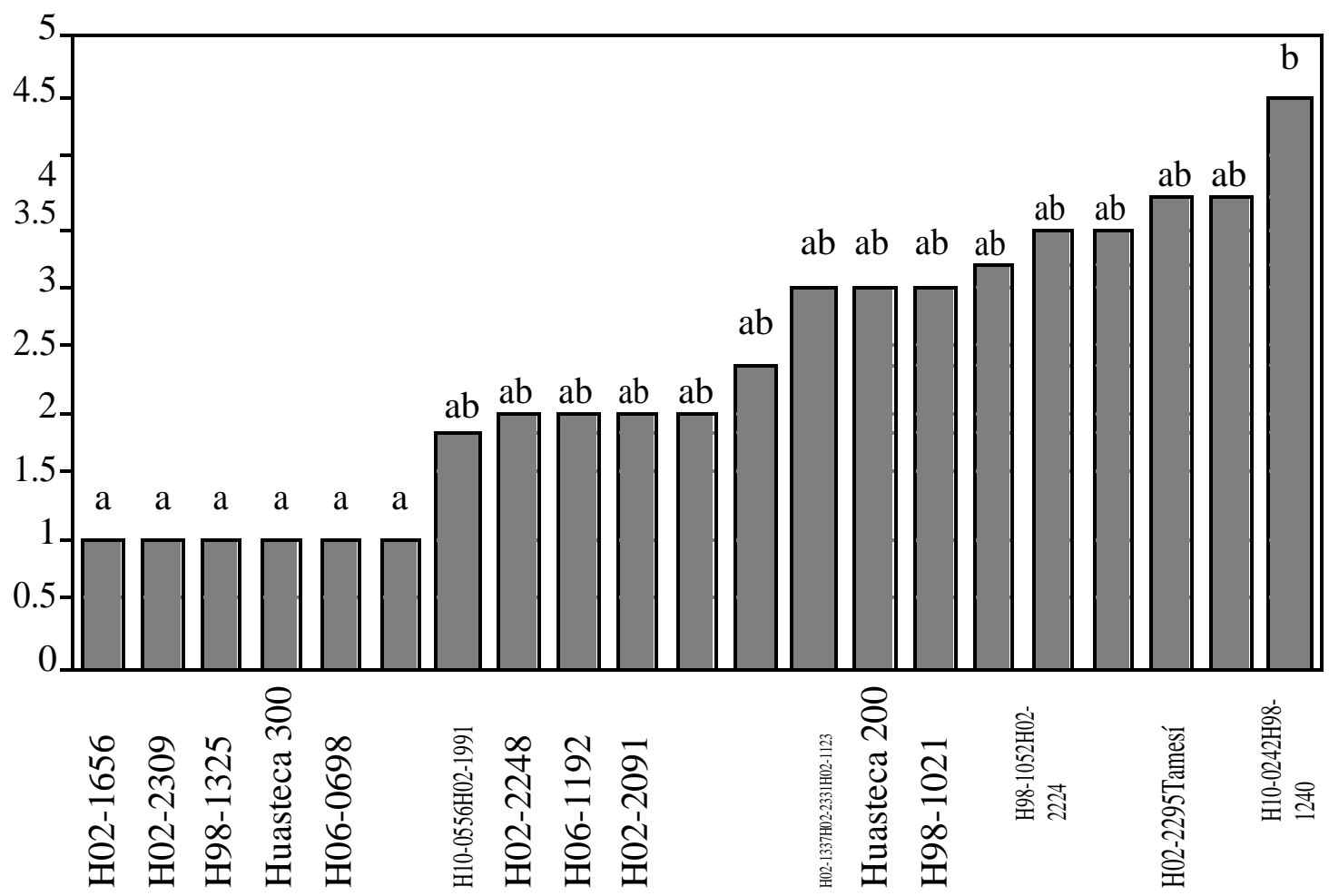

Figura 1. Grado de cierre de las hojas por efecto del calor $\left(50 \pm 1{ }^{\circ} \mathrm{C}\right)$ durante dos horas, en 21 genotipos de soya en estado de plántula. Medias con la misma letra entre genotipos no son diferentes.

Los movimientos foliares son frecuentes en leguminosas (Sosa et al., 2000), el cambio de ángulo del foliolo se debe a variaciones de turgencia en las células del pulvinus, una estructura especializada en la base del pecíolo, que generalmente están asociadas con la luminosidad del día y la noche. No obstante,

Björkman y Demmig-Adams (1994) mencionaron que el movimiento de los foliolos también responde a la temperatura. Taiz y Zeiger (2006) reportaron que las células ventrales y dorsales del pulvinus son las encargadas del movimiento de la hoja y están localizadas en zonas opuestas; cuando las células ventrales se encuentran turgentes las hojas están abiertas, de forma contraria, al estar las dorsales turgentes se provoca el cierre.

En cuanto a los efectos genéricos del calor sobre GM, se observaron diferencias altamente significativas $(\mathrm{p} \leq 0.01)$ entre genotipos y niveles de temperatura. Considerando que $\theta 4$ se aplicó después de $\theta 3$, $\theta 2$ y $\theta 1$ a las 24,48 y $72 \mathrm{~h}$ respectivamente, acumuló el mayor efecto y mostró el GM más alto con respecto a los otros niveles de temperatura (Figura 2). El aumento de GM fue gradual conforme se iban aplicando los tratamientos.

\section{Revista Científica Biológico Agropecuaria Tuxpan 4 (1) ISSN: 2007-6940}




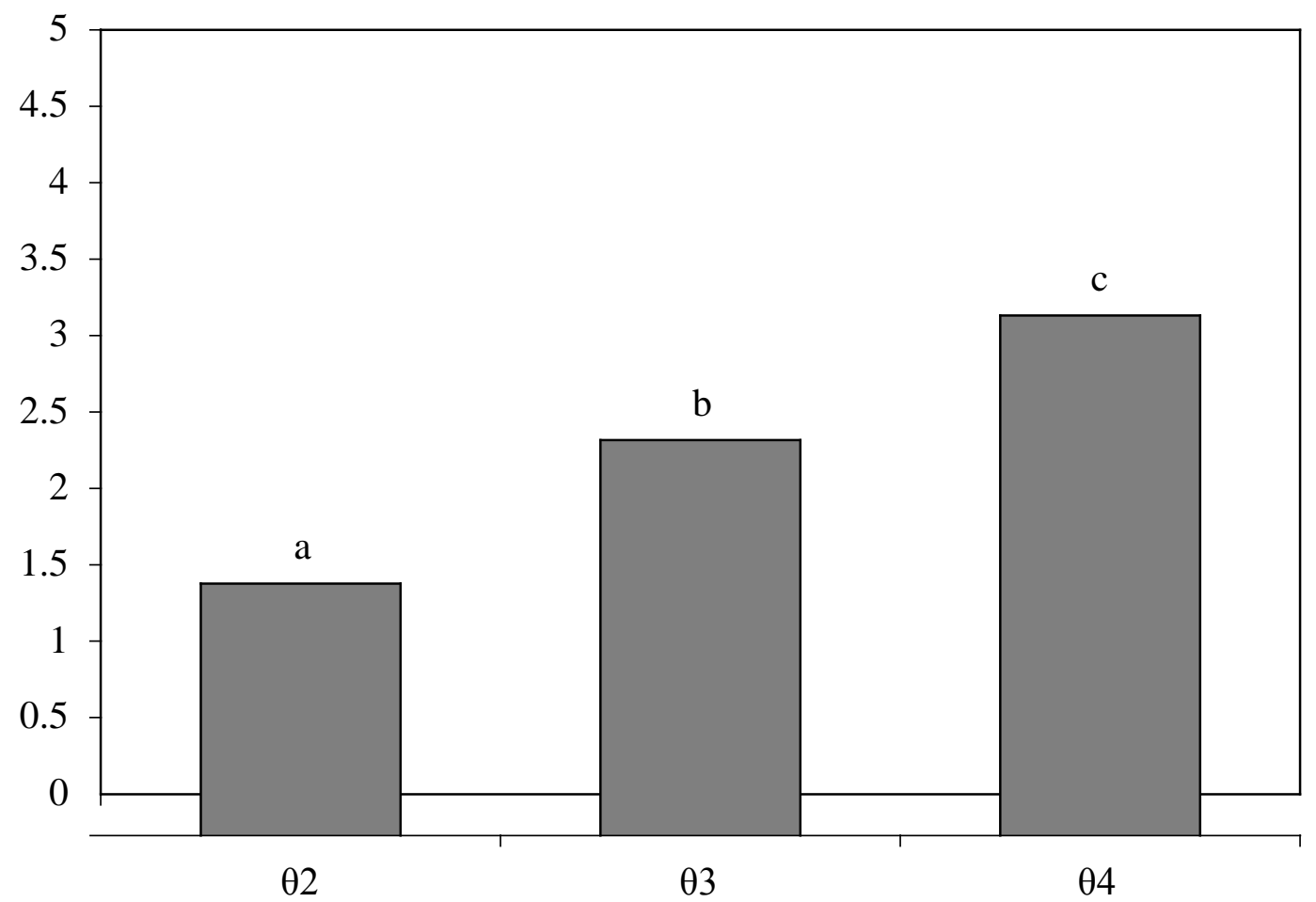

Figura 2. Efecto de tres niveles de temperatura acumulativos sobre el grado de marchitamiento de 21 genotipos de soya en estado de plántula. Medias con la misma letra entre niveles de temperatura no son diferentes. Tratamientos: $\theta 2=54 \pm 1{ }^{\circ} \mathrm{C} ; \theta 3$ $=58 \pm 1{ }^{\circ} \mathrm{C} ; \theta 4=62 \pm 1{ }^{\circ} \mathrm{C}$.

La relación entre el porcentaje de daños y el grado de temperatura aplicada a dos genotipos de soya, indicó que estos se incrementaron conforme aumentó el nivel de calor, mostrando los mayores daños entre los 55 y $60{ }^{\circ} \mathrm{C}$ (Martineau et al., 1979), resultados similares a los encontrados en el presente estudio, ya que la temperatura de $62 \pm 1{ }^{\circ} \mathrm{C}$ fue la que generó mayor GM en los genotipos evaluados.
Entre genotipos, GM fue más bajo en H02-1337 mostrando una mayor resistencia del tejido vegetal a los niveles de calor aplicados; H98-1240 fue el menos tolerante y acumuló mayor GM por efecto del estrés (Figura 3). Éste también fue el más sensible en $\mathrm{GCH}$ al exponerse a $\theta 1$, sin embargo la correlación entre GCH y GM no fue significativa. Lo que indica que son mecanismos relativamente diferentes. 


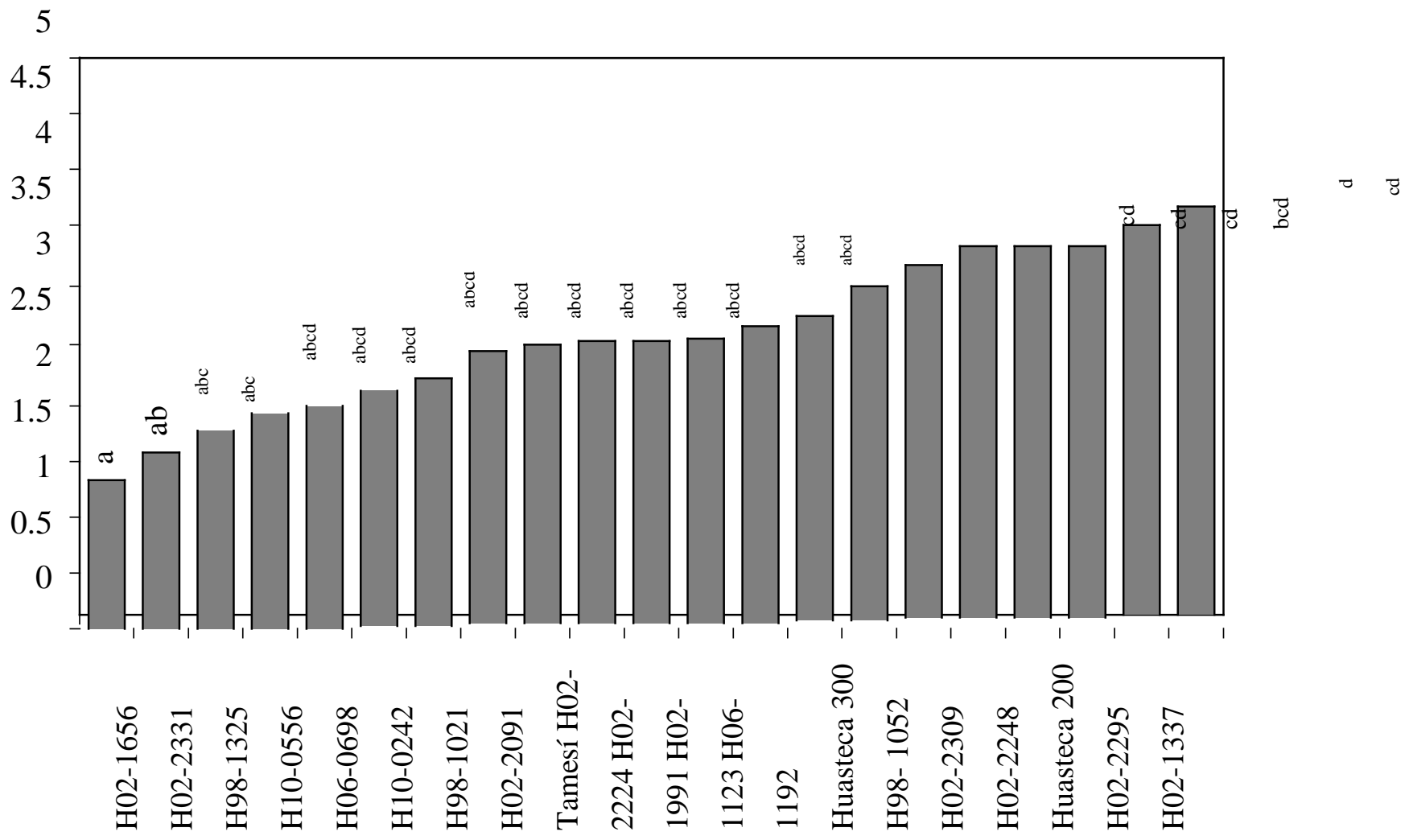

Figura 3. Grado de marchitamiento en 21 genotipos de soya sometidos a tres niveles de temperatura en etapa de plántula. Medias con la misma letra entre niveles de temperatura no son diferentes.

Sapra y Anaele (1991) también encontraron variabilidad genética en líneas de soya pertenecientes a diferentes grupos de madurez, las cuales se sometieron a regímenes de temperatura de $10,25,50$ y $110{ }^{\circ} \mathrm{C}$ en diferentes etapas del desarrollo; se identificaron las líneas PI 408.115, PI 423.827 B, PI 423.759 y Pershing como las más tolerantes al calor. Basados en la termoestabilidad de la membrana, Bouslama y Schapaugh (1984) reportaron $44 \%$ de daños en el cultivar de soya Essex y $75 \%$ en los cultivares: K1059, Cutler 71, Pixie, Cumberland y Bedford, cuando se sometieron a $50{ }^{\circ} \mathrm{C}$ durante el inicio de la floración.
Los genotipos con menor GM y que constituyen el $20 \%$ con mayor proporción de efectos genéricos de acuerdo con el modelo adaptado de Muñoz (1992) se observan en la Figura 4. El modelo indica que para conocer los efectos específicos al calor en este caso, se debe graficar la interacción genotipos por niveles de estrés en el $20 \%$ de los genotipos con mejores efectos genéricos, y aquéllos que no aumenten en gran proporción el GM al pasar del nivel más bajo al nivel más alto de temperatura, serán los que posean mejores características de resistencia. 


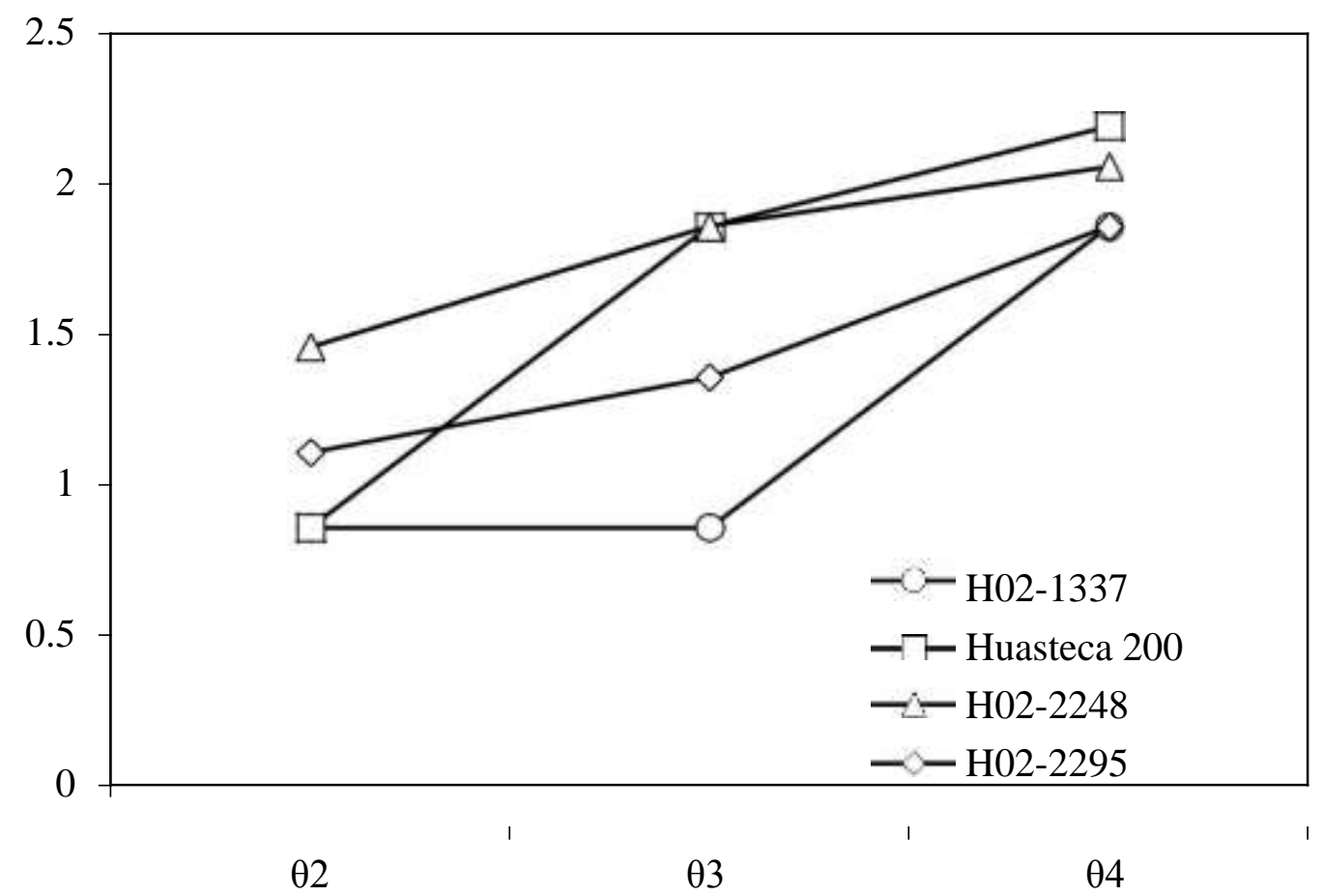

Figura 4. Interacción genotipos por niveles de temperatura sobre el grado de marchitez en genotipos de soya seleccionados por mayor proporción de efectos genéricos. Tratamientos: $\theta 2=54 \pm 1{ }^{\circ} \mathrm{C} ; \theta 3=58 \pm 1^{\circ} \mathrm{C} ; \theta 4=62 \pm 1{ }^{\circ} \mathrm{C}$.

La interacción mostró que H02-1337 no registró marchitamiento en $\theta 2$ y $\theta 3$, pero éste se incrementó de manera drástica al pasar de $\theta 3$ a $\theta 4$ (Figura 4). Por lo que se considera como resistente hasta los $58 \pm 1{ }^{\circ} \mathrm{C}$. Entre los niveles más altos de temperatura, la pendiente es menos ascendente en H02-2248 (Figura 4), identificándose como el genotipo con mayor proporción de efectos específicos a calor entre $58 \pm 1$ y $62 \pm 1{ }^{\circ} \mathrm{C}$.
La respuesta de los genotipos al riego de recuperación también mostró variabilidad, ya que hubo genotipos cuyas hojas se marchitaron en mayor grado por el efecto del calor y su GR fue bajo, en comparación con otros que sufrieron pocos cambios y su GR fue alto. Sin embargo, la variabilidad más bien puede atribuirse a efectos aleatorios y no al efecto del genotipo, ya que según la prueba de medias, el agrupamiento de genotipos fue muy estrecho (Figura 5). 


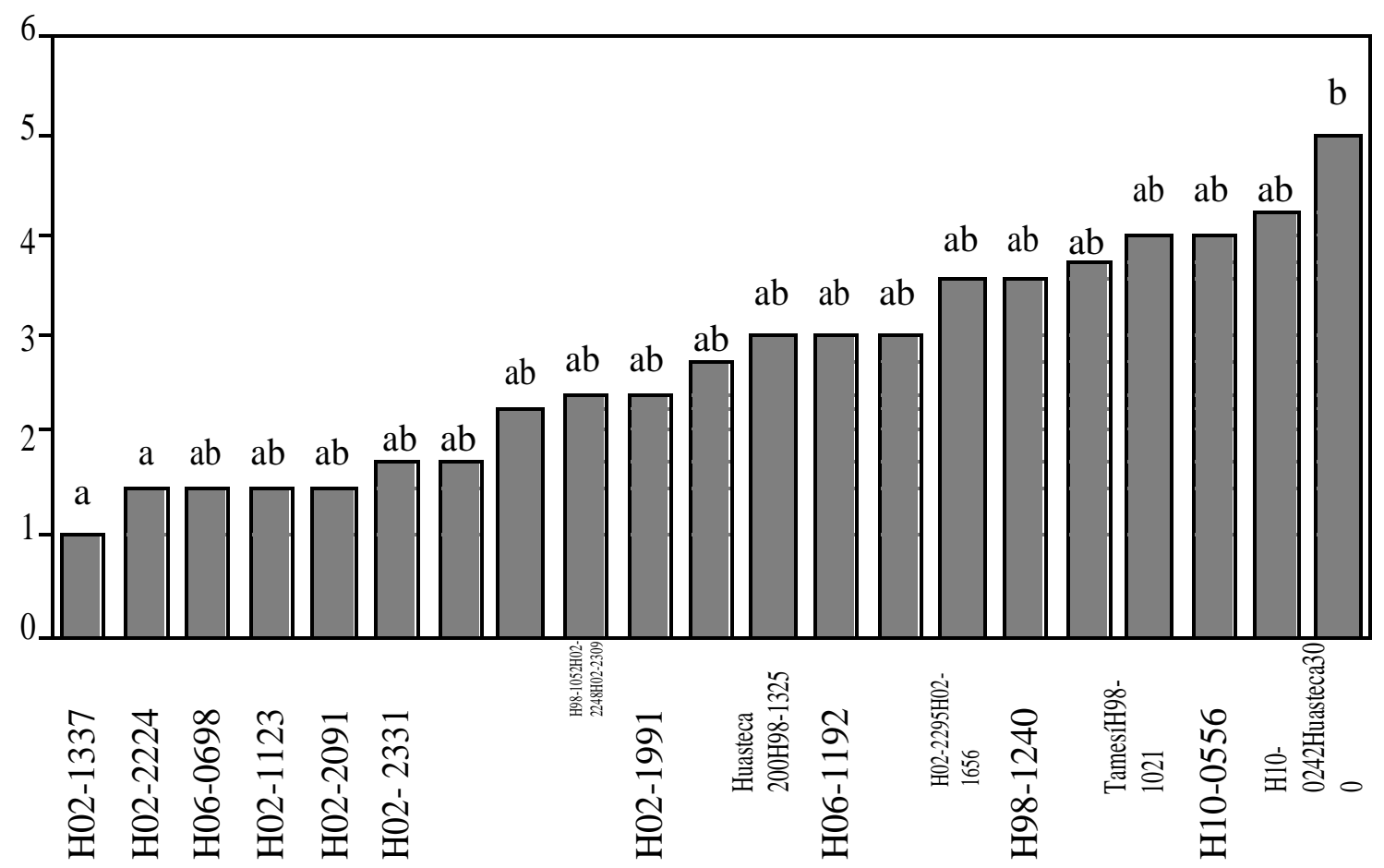

Figura 5. Efecto del riego sobre el grado de recuperación en genotipos de soya sometidos a varios tratamientos de calor. Medias con la misma letra entre genotipos no son diferentes.

Pueden considerarse a H02-1337 y H022224 como los que recuperaron en mayor proporción la turgencia y el verdor de las hojas a los tres días después del riego, pero estadísticamente formaron parte del grupo superior con otros 18 genotipos. Huasteca 300 obtuvo el GR más alto, indicando mayor susceptibilidad al calor, aunque sólo fue diferente de H012-1337 y H02-224 (Figura 5).

Los coeficientes de correlación entre $\mathrm{GCH}$, GM y GR no resultaron significativos. Por lo que los efectos de los tratamientos sobre las variables evaluadas fueron independientes.

\section{CONCLUSIONES}

La aplicación de $50 \pm 1{ }^{\circ} \mathrm{C}$ no causó marchitamiento, pero se observaron diferencias entre genotipos en el grado de cierre de las hojas: seis genotipos fueron totalmente insensibles y H98-1240 cerró sus hojas por completo como respuesta al calor. Entre niveles de temperatura, la aplicación de $62 \pm 1{ }^{\circ} \mathrm{C}$ causó el mayor marchitamiento, ya que acumuló los efectos de $50 \pm 1,54 \pm 1$ y $58 \pm 1$ ${ }^{\circ} \mathrm{C}$. Mientras que entre genotipos, 15 obtuvieron un bajo grado de marchitez, destacando H02-1337; H98-1240 resultó el más afectado por el estrés. De los genotipos seleccionados por efectos genéricos, H02-1337 no sufrió cambios al pasar de $54 \pm 1$ a $58{ }^{\circ} \mathrm{C} \pm$ 1 , considerándose como resistente en este nivel, y H02-2248 mostró mayor proporción de efectos específicos a la aplicación de $62 \pm 1{ }^{\circ} \mathrm{C}$. $\mathrm{La}$ respuesta de los genotipos al riego de recuperación indicó que hubo genotipos que se recuperaron más rápido y otros que tardaron más en hacerlo, sin embargo la variabilidad se debió más bien a efectos aleatorios. Los resultados anteriores sugieren que la selección de genotipos de soya resistentes a altas temperaturas por medio de la aplicación de aire caliente en estado de plántula, constituye una 
técnica sencilla y confiable con la cual se pueden obtener resultados en poco tiempo. Debido a que gran proporción de la variabilidad en los resultados fue aleatoria, se recomienda aumentar la unidad experimental y contemplar el uso de variables cuantitativas para reducir el error experimental.

\section{LITERATURA CITADA}

Allen, L. H., and K. J. Boote. 2000. Crop ecosystem responses to climate change: Soybean. In: K. R. Reddy and H. F. Hodges (eds.). Climate change and global crop productivity. $\mathrm{CAB}$ International, Wallingford, UK. pp. 133-160. https://doi.org/10.1079/9780851994390.01

Alexandrov, V. 1994. Functional aspects of cell response to heat shock. Int. Rev. Cytol. 148, 171-227. https://doi.org/10.1016/S007 Björkman, O., and B. Demmig-Adams. 1994. Regulation of photosynthetic light energy capture, conversion, and dissipation in leaves of higher plants. In: E. D. Schulze and M.M. Caldwell (eds.). Ecophysiology of photosynthesis. Springer-Verlag, Berlin. pp. 17-47. https://doi.org/10.1007/978-3-642-79354-7

Borthwick, H.A., and M. W. Parker. 1940. Floral initiation in Biloxi soybeans as influenced by age and position of leaf receiving photoperiodic treatment. Bot. Gaz. 101: 806-812. https://doi.org/10.1086/334916

Bouslama, M., and Schapaugh, W. T. 1984. Stress tolerance in soybeans. I. Evaluation of three screening techniques for heat and drought tolerance. Crop Sci. 24: 933-937. https://doi.org/10.2135/cropsci1984.00111

Gibson, L. R., and R. E. Mullen. 1996. Soybean seed quality reductions by high day and night temperature. Crop Sci. 36: 16151619. https://doi.org/10.2135/cropsci1996.00111

Lobell, D. B., and G. P. Asner. 2003. Climate and management contributions to recent trends in U.S. agricultural yields. Science 299: 1032.
Maldonado M., N., G. Ascencio L., G. Espinosa V., y M. de los A. Peña del R. 2013. Estrategias tecnológicas para contrarestar la sequía en la producción de soya en el sur de Tamaulipas. INIFAP. México. 65 p.

Martineau, J. R., Specht, J. E., Williams, J. H., and Sullivan, C. Y. 1979. Temperature tolerance in soybeans. I. Evaluation of a technique for assessing cellular membrane thermostability. Crop Sci. 19: 75-78. https://doi.org/10.2135/cropsci1979.001118 Mohammed, A. S., V. Gopal K., S. Koti, and K. Raja R. 2007. Pollen-bassed screening of soybean genotypes for high temperaturas. Crop Sci. 47: 219-231. https://doi.org/10.2135/cropsci2006.07.044 Muñoz O., A. 1992. Modelo uno o de interacción genotipo por niveles de sequía y resistencia a factores adversos. In: Memoria del Simposio Interacción Genotipo-Ambiente en Genotecnia Vegetal. 22-27 de marzo. Sociedad Mexicana de Fitogenética, Guadalajara Jalisco, México. pp. 261-266.

Peet, M. M. and Willits, D. H. 1998. The effect of night temperature on greenhouse grown tomato yields in warm climate. Agric. Forest Meteorol. 92: 191-202. https://doi.org/10.1016/S0168-1923(98)000

Raper, C. D., and P. J. Kramer. 1987. Soybeans: Improvement, production, and uses. In: J. R. Wilcox (ed.). Stress physiology. Monograph 16. ASA, CSSA, and SSSA, Madison, WI. pp. 589-641.

Sapra, V. T., and Anaele, A. O. 1991. Screening soybean genotypes for drought and heat tolerance. J. Agron. Crop Sci. 167: 96-102. https://doi.org/10.1111/j.1439-037X.1991.t 
Salem, M. A., Kakani, V. G., Koti, S., and Reddy, K. R. 2007. Pollen-based screening of soybean genotypes for high temperatures. Crop Sci. 47: 219-231. https://doi.org/10.2135/cropsci2006.07.044

Sosa E., Ortega, M., Escalante, A., Engleman, M., y González, V. 2000. Inclinación de láminas de frijol durante el día. Terra Latinoam. 18: 147-152.

Sullivan, C. Y. 1972. Mechanisms of heat and drought resistance in grain sorghum and methods of measurement. In: Rao, N. G. P., and L. R. House (eds.). Sorghum in Seventies. Oxford \& I.B.H. Publishing Co. New Delhi, India.

Taiz, L., y E. Zeiger. 2006. Fisiología Vegetal. Universitat Jaume I. Castellón de la Plana, España. 1336 p.

Thomas, J. M. G., K. J. Boote, L. H. Allen, M. Gallo-Meagher, and J. M. Davis. 2003. Elevated temperature and carbon dioxide effects on soybean seed germination and transcript abundance. Crop Sci. 43: 15481557.

https://doi.org/10.2135/cropsci2003.1548

Vu, J. C. V., L. H. Allen, K. J. Boote, and G. Bowes. 1997. Effects of elevated CO2 and temperature on photosynthesis and rubisco in rice and soybean. Plant Cell Environ. 20: $68-76$. https://doi.org/10.1046/j.1365-3040.1997.d Wahid, A., Gelani, S., Ashraf, M., and Foolad, M. R. 2007. Heat tolerance in plants: an overview. Environ. Exp. Bot. 61: 199-223. https://doi.org/10.1016/j.envexpbot.2007.0 
Copyright (c) 2016 Julio C. Garcia Rodriguez, Abel Muñoz Orozco, Nicólas Maldonado Moreno,

Ser afin Cruz Izquierdo y Guillerm o Ascencio-Luciano

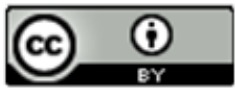

Este tex to está protegido por una licencia licencia Creative Commons 4.0.

Usted es libre para Compartir — copiar y redistribuir el material en cualquier medio o form ato- y Adaptar el documento —remezclar, transformar y crear a partir del material- para cualquier propósito, , incluso para fines comerciales, siempre que cumpla la condición de:

Atribución: Usted debe dar crédito a la obra original de manera adecuada, proporcionar un enlace a la licencia, e in dicar si se han realizado cambios. Puede hacerlo en cualquier form a razonable, pero no de form a tal que sugiera que tiene el apoyo del licenciante o 10 recibe por el uso que hace de la obra.

Resumendelicencia - Textocompletodelalicencia 